\title{
Safe medical staffing: the association between medical staff numbers and mortality in English hospitals
}

\author{
Authors: Phil Harvey* and Nigel Trudgill
}

\section{Background}

The Royal College of Physicians' Guidance on safe medical staffing (GoSMS) report proposes staff numbers per 30-bed medical ward for expert clinical decision makers (ECDM), senior clinical decision makers (SCDM) and competent clinical decision makers (CCDM). The report recognised that outcome data in relation to medical staffing levels are limited.

The Summary Hospital level Mortality Indicator (SHMI) is the adjusted measure of mortality calculated for hospital providers comparing actual with expected mortality. A SHMI < 1 implies lower mortality than expected. We have examined the relationship between mortality at a trust level and medical staffing.

\section{Methods}

The number of medical doctors by grade was accessed from electronic staff records provided by Health Education England. Medical bed numbers and SHMI by acute hospital trust was obtained from the NHSi Model Hospital resource. Medical beds per ECDM, SCDM and CCDM working in medical specialties were calculated. Only acute hospital trusts were included; community, mental health and specialist trusts were excluded. Univariate linear regression was undertaken with SHMI as the dependent variable to examine associations with medical staffing levels.

\section{Results}

A total of 136 trusts were included in the final analysis. The median number of medical beds was 391 (interquartile range (IQR) 273-489) per trust. The median numbers of ECDM, SCDM and CCDM were 53.7 (IQR 37.2-84.3), 22.5 (12.0-38.2) and 36.8 (27.6-59.2) respectively. The mean number of beds per ECDM, SCDM and CCDM were 7.2 (2.6), 19.3 (11.4) and 10.3 (4.1) respectively. Univariate linear regression analysis demonstrated highly significant associations between SHMI and ECDM $(0.015$ (95\% confidence interval 0.008-0.021), $p<0.001)$, SCDM (0.003 (0.001-0.005), $p=0.001)$ and CCDM (0.009 (0.004-0.014), $\mathrm{p}=0.001$ ).

Fitting a linear relationship between beds per staff member and SHMI, trusts are more likely to achieve SHMI $<1$ if, per 30-bed ward, a trust employs more than 4.3 ECDMs, 1.3 SCDMs and 2.9 CCDMs. GoSMS suggested a minimum of 1 ECDM, 1 SCDM and 2 CCDMs per 30-bed ward per week.

\section{Conclusions}

Higher numbers of all grades of medical staff per bed are associated with lower mortality. It is not possible to confer causality from this association but acute hospitals with higher staffing levels per bed appear to have lower than expected mortality. 